\title{
Effects of Electroacupuncture on the Regulation of Chemokine Induced Spinal Activation of Microglia in the Rat Model of Neuropathic Pain
}

\author{
Vishnumolakala Sindhuri ${ }^{1,3}$, Ji Eun Lee ${ }^{3}$, Hye-Ji Park ${ }^{3}$, So-Hee Kim ${ }^{3}$, Sungtae Koo ${ }^{1,2,3}$ \\ ${ }^{1}$ Department of Korean Medical Science, School of Korean Medicine, Pusan National University, \\ ${ }^{2}$ Division of Meridian and Structural Medicine, School of Korean Medicine, Pusan National University, \\ ${ }^{3}$ Korean Medicine Research Center for Healthy Aging, Pusan National University

\section{흰쥐 신경병증성 통증 모델에서 전침이 케모카인이 유도하는 척수 교세포 활성화 조절에 미치는 영향}

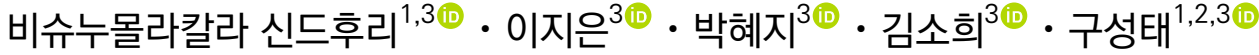 \\ '부산대학교 한의학전문대학원 한의과학과, ${ }^{2}$ 부산대학교 한의학전문대학원 경락구조의학부, ${ }^{3}$ 부산대학교 건강노화한의과학연구센터
}

\begin{abstract}
Objectives: Microglia play a crucial role in electroacupuncture (EA) analgesia on neuropathic pain. The role of chemokines in producing analgesic effects of EA, however, is largely unknown. In the present study, we investigated the role of chemokines in producing analgesic effects of EA in the neuropathic pain model. Methods : Sprague-Dawley rats were randomly assigned into three groups (anesthetized group (ANE), non-acupoint EA group (NAP), and ST36 - GB34 EA group (ACU)). Neuropathic pain was induced by tight ligation of L5 spinal nerve. Mechanical and thermal hypersensitivity of hind paw was tested. Western blot tests and immunofluorescence assay for C-C motif chemokine ligand 2 (CCL2) levels and microglia activation were performed on spinal cord L5/6. EA was treated once daily from the 3rd day after surgery for 5 days. Results : EA treatments applied to ST36 and GB34 significantly reduced both mechanical and thermal hypersensitivity after two and three times of treatment, respectively. While CCL2 expression significantly increased in neuropathic rats, it was significantly reduced in the ACU. In addition, co-localization of CCL2 and activated microglia significantly decreased in the ACU compared to those of ANE and NAP in the spinal cord L5/L6 dorsal horn. Conclusions: The present results suggest that EA applied to ST36 and GB34 modulates the reduction of CCL2 release from the injured neurons and consequently decreases microglia activation in the spinal cord. Regulation of chemokine induced spinal activation of microglia plays a key role in analgesic effects of EA in the rat model of neuropathic pain.
\end{abstract}

Key words : electroacupuncture, neuropathic pain, chemokine, microglia

Received November 8, 2019, Revised December 9, 2019, Accepted December 18, 2019

Corresponding author: Sungtae Koo

Department of Korean Medical Science and Division of Meridian and Structural Medicine, School of Korean Medicine, Pusan National University, 49

Busandaehak-ro, Mulgeum-eup, Yangsan 50612, Korea

Tel: +82-51-510-8474, Fax: +82-51-510-8439, E-mail: stkoo@pusan.ac.kr

This work was supported by the National Research Foundation of Korea (NRF) grant funded by the Korea government (MSIT) (No. 2017R1A2B4010250).

(C) This is an open access article distributed under the terms of the Creative Commons Attribution Non-Commercial License (http://creativecommons.org/licenses/ by-nc/4.0) which permits unrestricted non-commercial use, distribution, and reproduction in any medium, provided the original work is properly cited. 


\section{Introduction}

Neuropathic pain is defined as pain caused by a lesion or disease of the somatosensory nervous system ${ }^{11}$. Neuropathic pain hinders with quality of life and is often poorly managed, the reason being unclear mechanisms. Neuropathic pain can be caused by central or peripheral injuries ${ }^{2}$ and the nature and severity of neuropathic pain are relative to the injury. Chronic neuropathic pain caused by peripheral nerve is often associated with hyperalgesia and allodynia ${ }^{3}$. In the spinal cord, nerve injury induces the increase of excitatory synaptic transmission like N-methyl D-aspartate (NMDA) and $\alpha$-amino 3-hydroxy-5-methyl 4-isoxazolepropionic acid (AMPA) receptors and decreases the gamma-aminobutyric acid (GABA) receptor and glycine receptor mediate inhibitory synaptic transmission in dorsal horn neurons ${ }^{4-6}$. After the nerve injury, there is neuronal excitability due to activation of excitatory glutamate receptors. These effected neurons release chemokines like $\mathrm{C}-\mathrm{C}$ motif chemokine ligand 2 (CCL2) ${ }^{7}$. The CCL2 binds to its receptors CCR2 ${ }^{8)}$ on microglial cells leading to microglial activation. Recent evidences show that microglia play an important role in the development and maintenance of neuropathic pain". After the nerve injury, the microglia get activated in 3 days and helps in the development of neuropathic pain. The activated microglia release cytokines like Interlukin-1 $\beta$ (IL-1 $\beta$ ) and Tumor Necrosis Factor (TNF- $\alpha)^{10)}$.

Acupuncture is an important treatment technique of Asian traditional medicine which involves insertion of needles into certain points in the body. Electroacupuncture (EA) has been the recent choice of medicine as it has proven to be quite effective in treating patients suffering with various disorders particularly pain related diseases. Recent evidences showed that there is decreased activation of microglia ${ }^{11)}$ which helps in producing analgesic effects. The expression of BDNF has significantly reduced after EA treatment and also the BDNFTrkB pathway has been downregulated ${ }^{12)}$ after EA in neuropathic pain rat model. EA stimulation also helps in the inhibition of p38 mitogen-activated protein kinase (MAPK) and extracellular signal-regulated kinase (ERK) activation in micro- gliacytes at the L4- ${ }^{11)}$. Transient receptor potential vanilloid 1 (TRPV1) has also been down regulated after EA treatment in neuropathic pain models ${ }^{13}$. Several studies have also shown that EA activates somatostatin expression and glial cell line-derived neurotrophic factor (GDNF) proteins both in spinal dorsal horn and dorsal root ganglion, thereby producing analgesic effect of $\mathrm{EA}^{14,15)}$. However, the neurologic mechanism on which the EA acts to alleviate the neuropathic pain is still largely unknown.

To explore the underlying mechanism of EA analgesia, we hypothesized that chemokine CCL2 might be downregulated resulting in reduction of microglia activation and thus plays an important role in EA analgesia in the neuropathic pain. The aim of present study is to investigate the effects of EA on the modulation of chemokine expression and microglial activation in the spinal cord of neuropathic rats.

\section{Materials and Methods}

\section{Experimental animals}

Experiments were performed on 35 male Sprague Dawley rats. Animals were acclimatized to standard laboratory conditions i.e. reversed light dark cycle (12-hour dark and 12hour light cycle). They were housed in groups of three and were given free access to food and water ab libitum. All experimental procedures were performed according to the Animals (Scientific Procedures) Act 2008 (Korea) and compiled with the recommendations of the National Institute of Health's Guide for the Care and Use of Laboratory Animals. The studies were approved by the Ethics Committee on Animal Research of Pusan National University (PNU-20192370). All efforts were taken to minimize the number of animals utilized.

\section{Experimental procedures}

Twenty-one rats were randomly divided into three groups: the anesthetized group (ANE, $n=7$ ), group of EA applied to non-acupoint (NAP, n=7), group of EA applied to both of ST36 and GB34 points group (ACU, $n=7$ ). Fourteen animals 
were used to collect spinal cord samples of naïve (Naïve, $n=5$ ) and spinal nerve ligation model (SNL, $\mathrm{n}=9)$ ) animals. After baseline measurement tests on day 0 , the animals received neuropathic surgery. From day 3, EA was given every day for 5 days. Prior to the EA treatment, daily baseline values of behavioral tests were taken. In each group, L5-6 spinal cord of the rats were removed to analyze CCL2 and microglia using western blot and immunofluorescence stain on day 8 .

\section{Neuropathic surgery (Spinal Nerve Ligation, SNL)}

Rats were anesthetized with isoflurane (3\% induction, 1.5\% maintenance) mixed with $\mathrm{N}_{2} \mathrm{O} / \mathrm{O}_{2}$, then the $\mathrm{L} 5$ spinal nerve was tightly ligated as described before ${ }^{16}$. Briefly, a midline skin incision was made on the back at the lower lumbar region, the paraspinal muscles were retracted, and the left transverse process of the L5 vertebra was removed under the dissection microscope. The left L5 spinal nerve was identified and gently separated from the adjacent L4 spinal nerve; it was then tightly ligated using 6-0 silk thread. The wound was cleaned with saline, closed with wound clips, and rats were returned to their cages after recovering from anesthesia.

\section{EA Treatment}

EA was administered for 30 minutes at $2 \mathrm{~Hz}$ and $1 \mathrm{~mA}$ with $1 \mathrm{~ms}$ pulse width. In detail, EA was given under gaseous isoflurane anesthesia (3\% induction and 1.5\% maintenance). Stainless steel acupuncture needles $(0.25 \mathrm{~mm}$ diameter and $40 \mathrm{~mm}$ long) were inserted at ST36 (Joksamli) and GB34 (Yangneungcheon), as they are known for their analgesic effect ${ }^{17)}$. The acupuncture needles were stimulated using a Pulse Master Multi-Channel Stimulator SYS-A 300 (World Precision Instruments, Inc., Berlin, Germany). EA was applied at two different points on the contralateral hind paw to the surgery site. The first point was the Joksamli point (ST36) which is located $5 \mathrm{~mm}$ beneath the capitilum fibulae and posteriolateral to the knee joint and the second point was the Yangneungcheon point (GB34) which is $5 \mathrm{~mm}$ superiolateral to ST36. In the non-acupoint group, the needles were inserted on either side of the tail subcutaneously.

\section{Behavioral tests}

Mechanical allodynia was assessed using a Dynamic Plantar Anesthesiometer (Ugo Basile, Varese, Italy) by measuring the latency to withdraw the hind paw from a graded force applied to the plantar surface of the effected hind paws using a Von Frey filament. The cutoff force was set at $50 \mathrm{~g}$. Following acclimatization and 'after cessation of exploratory behavior, the operator placed the touch stimulator unit under the animal's paw, using the adjustable angled mirror to position the filament below the target area of the paw. After pressing the "start" key, an electrodynamic actuator of proprietary design lifted a straight metal filament, which touched the plantar surface and began to exert an increasing upward force at a preset rate of application until a stop signal (when the animal removed the paw) was attained. The paw withdrawal threshold was numerically shown in grams on the digital screen.

Thermal hyperalgesia was assessed by the method of Hargreaves et al. (1988) by measuring the latency to withdraw the hind paw from a focused beam of radiant heat (thermal intensity: infrared 3.0) applied to the plantar surface of the effected limb, using a commercial apparatus (Ugo Basile, Varese, Italy). The cutoff time was set at $20 \mathrm{~s}$. To measure rat hind paw thermal thresholds, each rat was placed in a clear plastic compartment of an eight-compartment box with a glass floor and allowed to acclimatize for 10 20 min. A paw-flick response was elicited by a high intensity infrared beam focused on the plantar surface of effected paws (Ugo Basile, Varese, Italy). The time that elapsed before reflex removal of the hind paw from the beam (paw withdrawal latency, PWL) was monitored. PWL was defined as the mean of three measurements at inter stimulus interval of five minutes. Rats were trained for at least three days in order for the variability between these trials to be approximately $0.5 \mathrm{~s}$.

\section{Western blot}

The animals were euthanized under pentobarbital anesthesia (intraperitoneal). Fresh samples of L4-L6 spinal cords were extracted. The samples were excised for protein extraction. 
Proteins were prepared by homogenizing the samples in a lysis buffer. The supernatants were collected into fresh tubes. The total protein concentrations were calculated using Bradford assay. 40 micrograms of protein extracts were subjected to electrophoresis using Bio-Rad Mini PROTEAN Tetra System (USA) and transferred to nitrocellulose membrane. The membrane was blocked using 5\%albumin in Tris-buffer saline, $0.1 \%$ Tween 20 (TBST), incubated with primary antibody: (CCL2 (Thermofischer, Waltham, Massachusetts, USA \#PA534505 1:500) in TBST with 3\% albumin and incubated at $4{ }^{\circ} \mathrm{C}$ overnight. The membranes were washed with TBST and incubated with secondary antibody (abcam, Eugene, Oregon, USA \#ab205718 1:2000) in TBST with 3\% albumin at room temperature for $11 / 2 \mathrm{hr}$. The membranes were again washed with TBST. Immunoreactive bands were detected using CCD imaging device (Image Quant LAS 4000, Fujifilm, Tokyo, Japan). The band intensities were analyzed using Image J software (National Institutes of Health, Bethesda, Maryland,USA).

\section{Immunofluorescence staining}

The animals were euthanized under pentobarbital intraperitoneal anesthesia. Fresh samples of L4-L6 spinal cords were extracted. The samples were sectioned using a cryostat (CM3050S, Leica biosystems, Wetzlar, Germany) and were stored at $-80^{\circ} \mathrm{C}$. The slides were selected and washed with PBS solutions. Unnecessary antibodies were blocked using CAS block (Invitogen-Molecular Probes, Inc., Camorilla, CA, USA) for $15 \mathrm{~min}$. The slides were incubated with MCP-1 polyclonal antibody (Thermofischer, Waltham, Massachusetts, USA \#PA5-34505) CCL2 marker at a dilution of 1:50 or Iba-1monoclonal antibody (Thermofischer, Waltham, Massachusetts, USA \#MA5-27726) microglial marker at a dilution of 1:200 and kept at $4{ }^{\circ} \mathrm{C}$ overnight. The samples were washed with Phosphate buffered saline, 0.1\% Tween 20 (PBST) solution and then incubated with Goat Anti-Rabbit IgG H\&L Alexa Fluor 594 (abcam, Eugene, Oregon, USA \#ab150080) at a dilution of 1:500 and Goat Anti-Mouse IgG H\&L Alexa Fluor 488 (abcam, Eugene, Oregon, USA \#ab150113) at a dilution of 1:500 respectively for $2 \mathrm{hrs}$ in a dark room. The samples were washed with PBST and incubated with DAPI for 30min. The samples were finally washed with PBST and mounted with a vector shield. The CCL2 and microglia were identified using fluorescence microscope (Image M1, Axio, Zeiss, Oberkochen, Germany). Later the microglial cells, CCL2 immunoreactive spots and merged cells were counted using i-solution software (Image and Microscope Technology, Daejeon, Korea).

\section{Statistical analysis}

The data of results are presented as mean \pm Standard Error of Mean (SEM). Statistical significance of differences between groups in behavioral tests were performed by two-way ANOVA followed by Bonferroni post hoc test. Statistical difference of western blot and immunofluorescence stain to compare SNL versus naïve animals was performed by t-test and that of ANE, NAP, and ACU were compared by one-way ANOVA followed by Duncan's post hoc test using Systat Sigma Plot Version 12.2. $p$-value $(p<0.05)$ were considered statistically significant.

\section{Results}

\section{Effects of EA on mechanical allodynia and thermal hyperalgesia}

All the animals developed mechanical sensitivity from day 3 after SNL surgery. The ANE animals remained to have low paw withdrawal thresholds (PWT) for 8 days. PWT were significantly improved in ACU group when compared to ANE and NAP animals from day 5 after SNL surgery. There is also a significant difference in PWT between NAP and ANE groups (Fig. 1).

All the animals developed thermal sensitivity from day 3 after SNL surgery. The ANE and NAP animals remained to have low paw withdrawal latency (PWL) for 8 days. PWL were significantly improved in ACU group when compared to ANE and NAP animals from day 6 after SNL surgery. There is no significant difference in PWL between NAP and ANE groups (Fig. 2). 


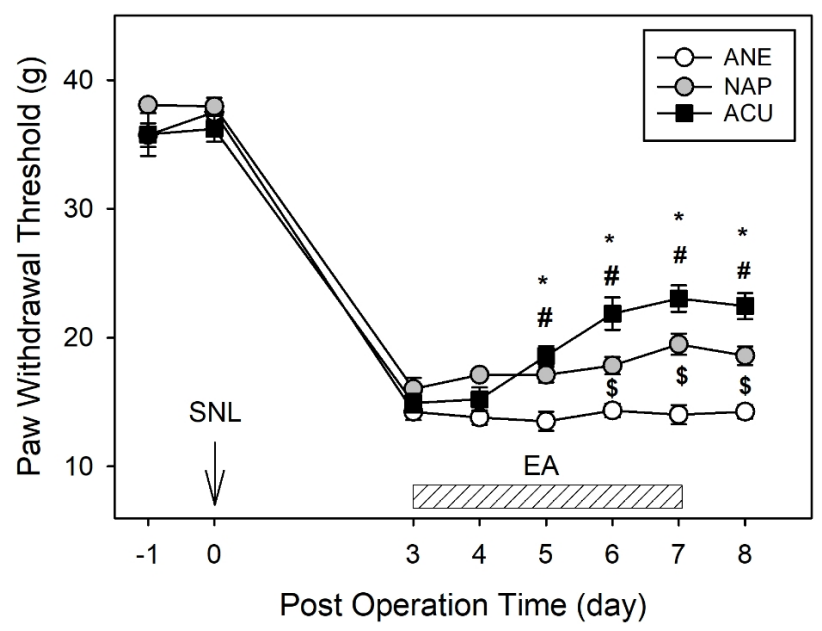

Fig. 1. Effect of EA on mechanical allodynia.

EA was given once a day to the neuropathic pain models from post-operative day 3 for five days. Mechanical allodynia was evaluated on neuropathic pain rat models. ANE, NAP and ACU group animals developed pain behavior from 3rd day after the SNL surgery. The EA treated animals slowly started to recover from pain from day 5 . ${ }^{*} p<0.05$ ANE vs ACU. ${ }^{\#} p<0.05$ NAP vs ACU. $\$ p<0.05$ ANE vs NAP. Each group is a mean of 7 animals.

\section{Effects of EA on CCL2 expression and microglia activation}

Based on western blot analysis, the concentration of CCL2 was increased significantly in SNL animals when compared to näve animals. According to immunofluorescence staining, the CCL2 expression was significantly increased in SNL animals compared to naïve animals. The microglial expression was also increased significantly in SNL animals compared to naïve animals (Fig. 3).

The expression of CCL2 in ACU animals has drastically decreased when compared to both ANE and NAP animals. Correlating with the western blot results, the immunofluorescence results showed a significantly decreased expression of CCL2 in ACU animals compared to both ANE and NAP animals. The expression of microglia was decreased in ACU animals with a statistical difference compared to ANE (Fig. 4).

\section{Discussion}

Neuropathic pain is most debilitating disorder and causing inconvenience to the human lifestyle. The drugs like NSAID'S,

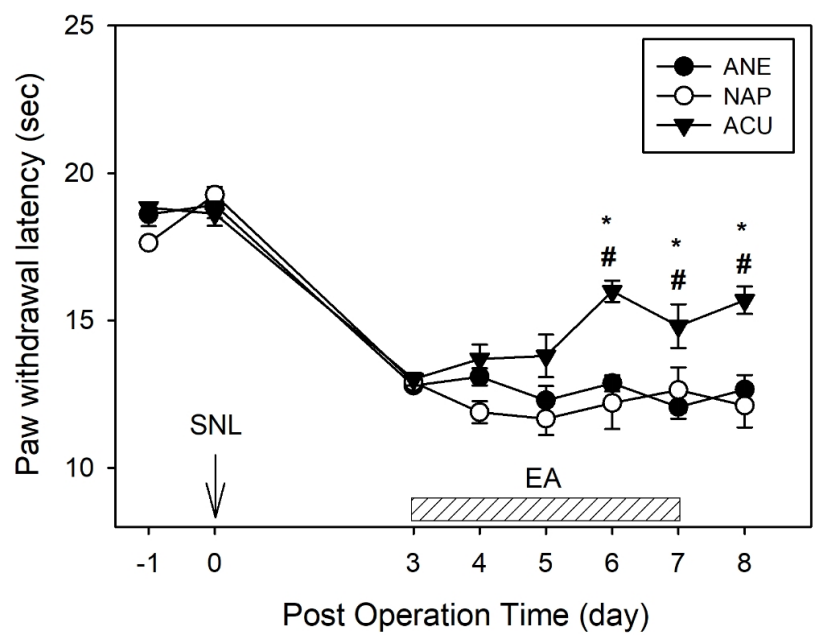

Fig. 2. Effect of EA on thermal hyperalgesia.

EA was given once a day to the neuropathic pain models from post-operative day 3 for five days. Thermal hypersensitivity was measured on neuropathic pain rat models. ANE, NAP and ACU group animals developed pain behavior from 3rd day after the SNL surgery. The EA treated animals slowly started to recover from pain from day 6 . There is statistically no significant difference between ANE and NAP groups. ${ }^{*} p<0.05$ ANE vs ACU. ${ }^{\#} p<0.05$ NAP vs ACU. Each group is a mean of 7 animals.

opioids though are being used as a line of treatment in relieving pain, they are resulting in various other side effects. Acupuncture is a form of ancient medicine where stainless steel needles are inserted into the bodies. This type of treatment is gaining popularity all over the world due to its fewer side effects. EA has also created wonders in relieving pain symptoms particularly in neuropathic pain disorders. Previous studies proved that reduced microglial activation played a vital role in producing analgesic effects in EA treated animals. Microglia are the first cells to be activated and remain activated following nerve injury ${ }^{18}$. The activated microglial cells remain active for several weeks ${ }^{19,20}$. CCL2 are present in small and medium sized spinal and DRG neurons ${ }^{21)}$. The role of chemokine CCL2 are still largely unknown. In our present study we examined the relation between microglia and chemokines in electro acupuncture treated neuropathic pain models.

Thus, in pain mechanism, after nerve injury the excited neurons release CCL2 which binds to its receptors CCR2 $2^{7)}$ on microglial cells. The ATP released from the damaged neurons bind to its receptor P2X4 on microglial cells ${ }^{22-24}$. This helps 

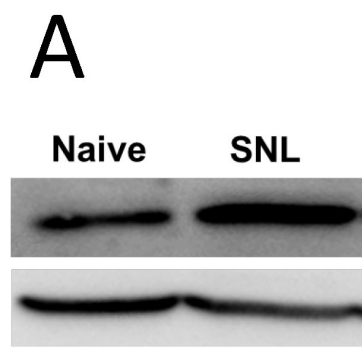

CCL2

$\beta$-Actin

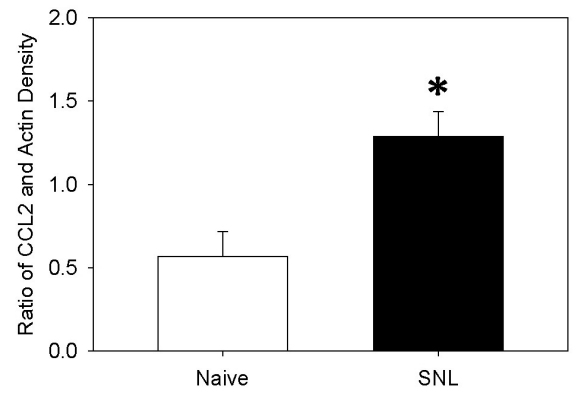

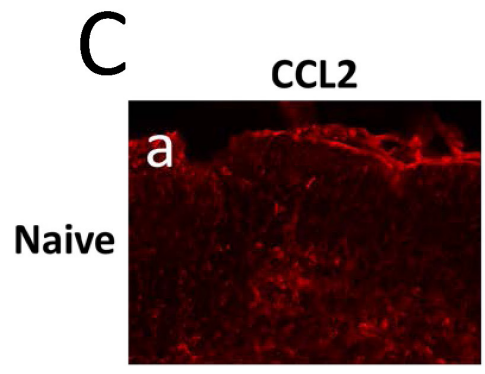
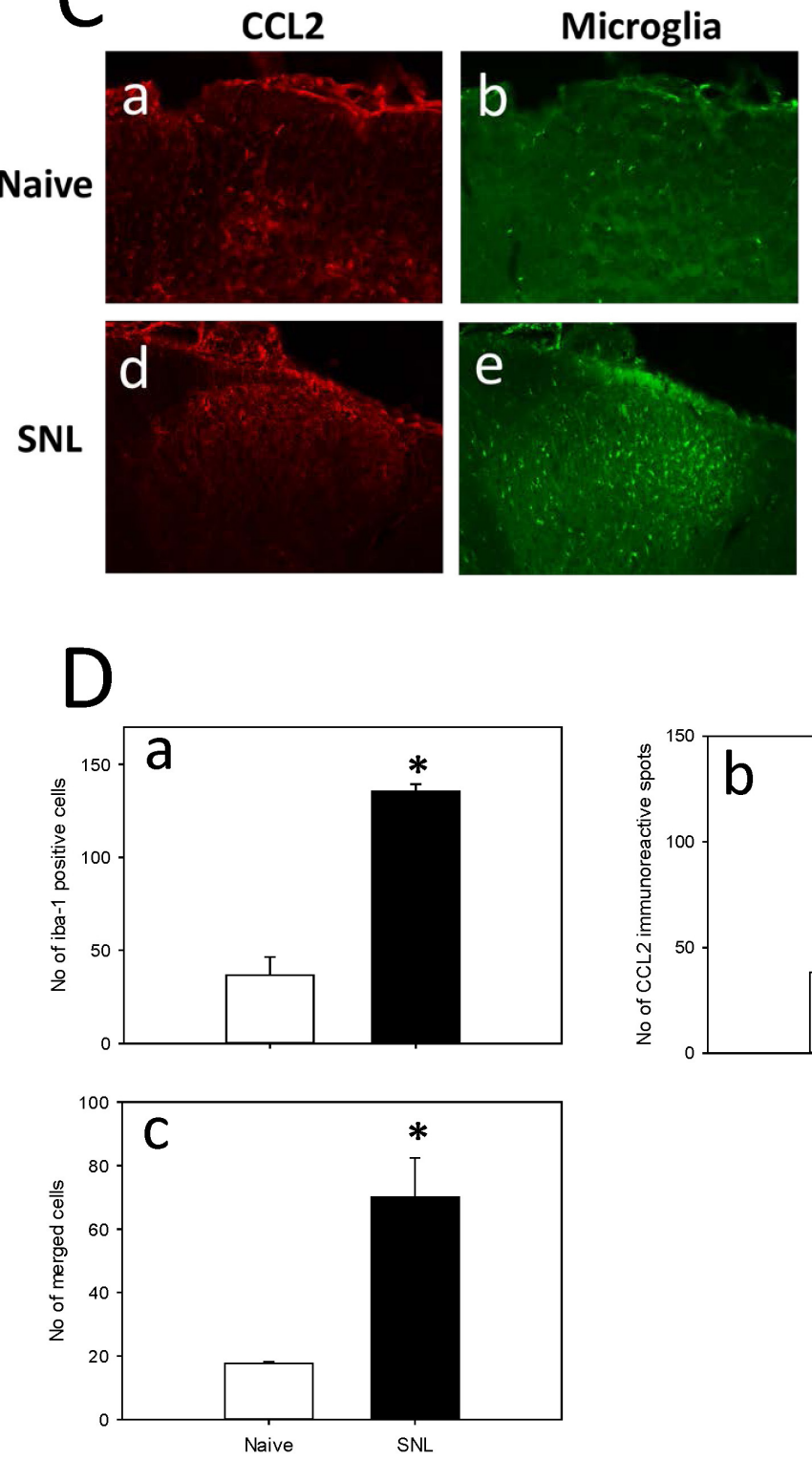
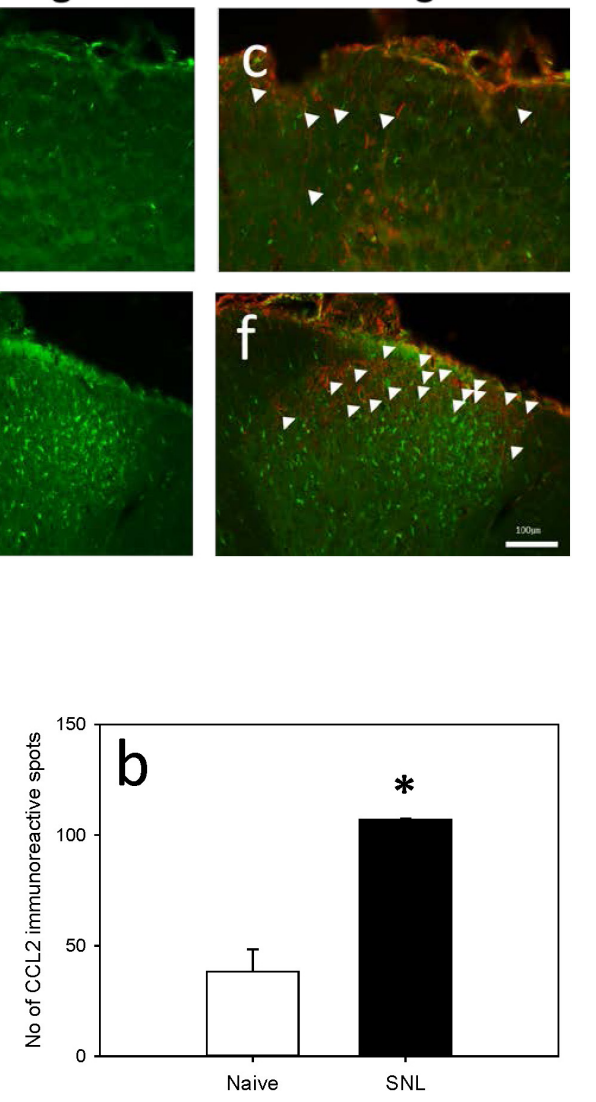

Fig. 3. Expression of $\mathrm{CCL} 2$ and microglia in Naïve and SNL animals. (A) Western blot analysis of L5/6 ipsilateral spinal cord was performed on day 8 after SNL surgery. CCL2 expression has been increased in SNL animals compared to naïve animals. (B) The SNL animals showed increased expression of CCL2 compared to naïve animals. Data are presented as Mean \pm SEM of the ratio of area of CCL2 to area of actin. *indicates values that are significantly different from the Naiive group. Immunofluorescence analysis of L5/ 6 ipsilateral spinal cord was performed on day 8 after SNL surgery. (C) CCL2 expression has been statistically increased in SNL animals compared to naïve animals (a and d). The activation of microglia (Iba-1; microglial marker) was more evident in the SNL animals when compared to naïve animals ( $b$ and $e$ ). The merged cells were also increased in SNL animals compared to Naïve animals (c and f). (D) SNL group showed increased number of both microglial cells and CCL2 expression with a statistical difference to the Naïve group. Data are presented as MEAN \pm SEM of number of lba-1 positive cells (a), number of CCL2 immunoreactive spots (b), number of merged cells (c). *indicates values that are significantly different from the Naivve group $(p<0.05)$. Scale bar is $100 \mu \mathrm{m}$. Arrow head indicates the co-localized cells. 
A

\section{ANE NAP ACU}

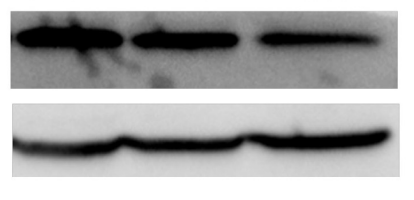

CCL2

$\beta$-Actin

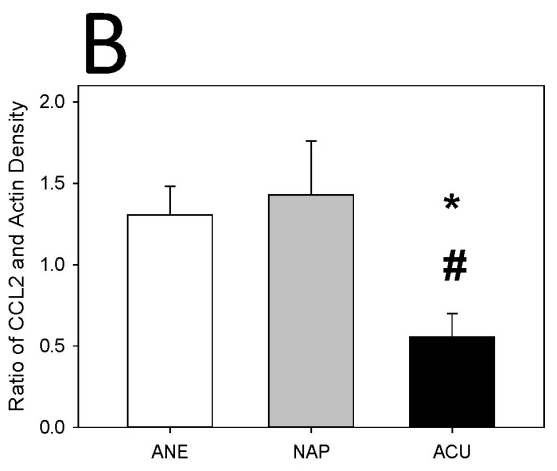

C
Microglia
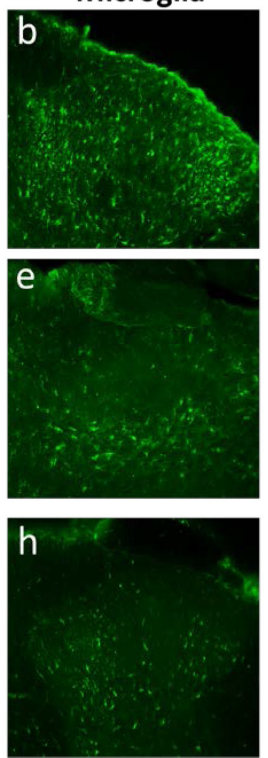
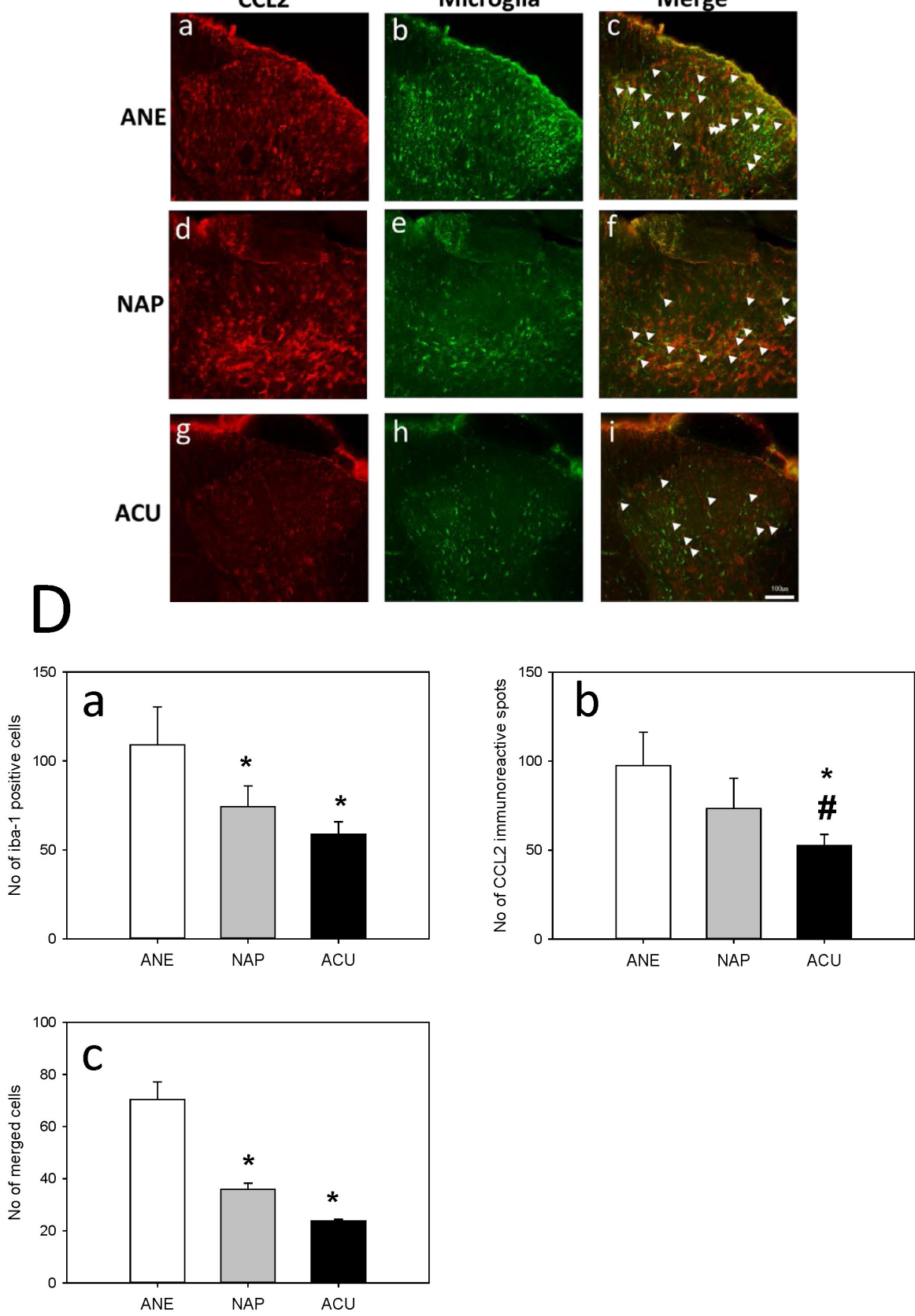

Fig. 4. Effect of EA on CCL2 expression and microglial activation in neuropathic animals.

(A) Western blot analysis of L5/6 ipsilateral spinal cord was performed on day 8 after SNL surgery. CCL2 expression has been decreased in ACU animals compared to ANE animals. (B) The ACU animals showed increased expression of TNF $\alpha$ compared to ANE animals. Data are presented as Mean \pm SEM of the ratio of area of CCL2 to area of actin. *indicates values that are significantly different from the ANE animals. Immunofluorescence analysis of L5/6 ipsilateral spinal cord was performed on day 8 after SNL surgery. (C) CCL2 expression in ANE (a), NAP (d), ACU (g) groups. The activation of microglia (Iba-1; microglial marker) was shown in ANE (b), NAP (e), ACU (h) animals. The merged cells were shown in ANE (c), NAP (f), ACU (i) animals. (D) ACU animals showed decreased number of both microglial cells and TNF $\alpha$ expression with a statistical difference to the ANE animals. Data are presented as MEAN \pm SEM of number of Iba-1 positive cells (a), number of CCL2 immunoreactive spots (b), number of merged cells (c). * and \# indicate values that are significantly different from the ANE and NAP groups respectively $(p<0.05)$. Scale bar is $100 \mu \mathrm{m}$. Arrow head indicates the co-localized cells. 
in microglial activation. The activated microglia release various mediators like $\mathrm{BDNF}^{4}$, proinflammatory cytokines like IL-1 $\beta$ and TNF $\alpha^{10)}$. These mechanisms further help in the development and maintenance of pain.

The results of this study show a significant difference between Naïve and SNL groups and also a notable difference between ANE and ACU groups. In the behavioral tests there are increased pain thresholds and latencies in all the three groups after SNL surgery. The mechanical thresholds have declined notably not only in ACU animals but also NAP animals when compared to ANE animals. There is also a significant difference between the NAP and ACU groups. PWL have reduced significantly in ACU animals when compared to ANE and NAP animals. In thermal hyperalgesia tests, there is no significant difference between ANE and NAP animals. This shows that the animals have recovered from pain after EA treatment for 5 days.

The expression of CCL2 has increased significantly in the SNL group compared to the Naivve group in western blot analysis. The western blot results showed that CCL2 expression has decreased statistically in EA treated group compared to both ANE and NAP groups. The expression of microglia has increased remarkably in the SNL group compared to Naïve animals and had a downfall in EA treated group compared to ANE animals. The microglial expression has declined with a significant difference in NAP animals also compared to ANE animals. The immunofluorescence results suggest an increased expression of CCL2 in SNL animals compared to Naivve animals and decreased expression of CCL2 in ACU animals compared to ANE animals. There is also a significant difference between the NAP and ACU group animals. The co-localization of CCL2 and microglia have also seen steep increase in SNL animals when compared to naïve animals. Interestingly, co-localization of CCL2 and microglia have declined with a statistical difference not only in ACU animals but also in NAP animals compared to ANE animals. This results are consistent with those obtained from mechanical allodynia tests. The decreased expression of CCL2 in NAP group shows that EA stimulation at points that are not on the meridians also show a mild analgesic effect but are not as prominent as those elucidated by points on the meridians. Based on this result CCL2 and microglia are interrelated and play a major role in producing analgesic effects in electro acupuncture analgesia.

Previous studies have already proved that reduced microglial activation plays a major role in producing analgesic effects after EA treatment ${ }^{11}$. Based on our study, CCL2 expression has declined in EA treated group. Hence, we hypothesized that electro acupuncture helps in the reduction of CCL2 expression which helps in reducing the microglial activation. This mechanism helps in producing the analgesic effects of electro acupuncture. Thus, chemokine CCL2 plays a major role in producing analgesic effects in EA. The expression of CCR2 and differentiation of activated microglia in the total count of microglia would have further enhanced the study. Further studies are needed to know more clear underlying mechanisms to understand the analgesic effect of EA in neuropathic pain models. The role of cytokines and its connection to chemokines and microglia can be further studied.

\section{Conclusion}

Our data showed that repeated EA treatment resulted in producing analgesic effects in neuropathic pain animal models. Our results suggest that EA applied to ST36 and GB34 modulated the reduction of CCL2 release from the injured neurons and consequently decrease microglial activation in the spinal cord. Thus, regulation of chemokine induced spinal activation of microglia plays a key role in producing analgesic effects in EA treated neuropathic pain models. However, further studies have to be done to understand the clear mechanism of analgesic effect of electro acupuncture in neuropathic pain rat models.

\section{Acknowledgement}

This work was supported by the National Research Foun- 
dation of Korea (NRF) grant funded by the Korea government (MSIT) (No. 2017R1A2B4010250).

\section{Funding}

None.

\section{Data availability}

The authors can provide upon reasonable request.

\section{Conflicts of interest}

The authors have declared that no conflicts of interest exists.

\section{References}

1. Adler JE, Nico L, VandeVord P, Skoff AM. Modulation of neuropathic pain by a glial-derived factor. Pain Med. 2009 ; 10 : 1229-36.

2. Attal N, Fermanian C, Fermanian J, Lanteri-Minet M, Alchaar H, Bouhassira D. Neuropathic pain: are there distinct subtypes depending on the aetiology or anatomical lesion? Pain. 2008; 138 : 343-53.

3. Baron R. Neuropathic pain: a clinical perspective. Handb Exp Pharmacol. 2009 : 3-30.

4. Coull JA, Beggs S, Boudreau D, Boivin D, Tsuda M, Inoue K, et al. BDNF from microglia causes the shift in neuronal anion gradient underlying neuropathic pain. Nature. 2005 ; 438 : 1017-21.

5. Ji RR, Kohno T, Moore KA, Woolf CJ. Central sensitization and LTP: do pain and memory share similar mechanisms? Trends in neurosciences. $2003 ; 26: 696-705$.

6. Woolf CJ, Mannion RJ. Neuropathic pain: aetiology, symptoms, mechanisms, and management. Lancet (London, England). 1999; $353: 1959-64$
7. Dansereau MA, Gosselin RD, Pohl M, Pommier B, Mechighel P, Mauborgne A et al. Spinal CCL2 pronociceptive action is no longer effective in CCR2 receptor antagonist-treated rats. Journal of neurochemistry. $2008 ; 106: 757-69$.

8. Zhang ZJ, Jiang BC, Gao YJ. Chemokines in neuron-glial cell interaction and pathogenesis of neuropathic pain. Cellular and molecular life sciences : CMLS. 2017 ; 74 : 3275-91.

9. Tu W, Wang W, Xi H, He R, Gao L, Jiang S. Regulation of Neurotrophin-3 and Interleukin-1beta and Inhibition of Spinal Glial Activation Contribute to the Analgesic Effect of Electroacupuncture in Chronic Neuropathic Pain States of Rats. Evidence-based complementary and alternative medicine : eCAM. 2015 ; 2015 : 642081.

10. Lee HL, Lee KM, Son SJ, Hwang SH, Cho HJ. Temporal expression of cytokines and their receptors mRNAs in a neuropathic pain model. Neuroreport. $2004 ; 15: 2807-11$.

11. Choi DC, Lee JY, Lim EJ, Baik HH, Oh TH, Yune TY. Inhibition of ROS-induced p38MAPK and ERK activation in microglia by acupuncture relieves neuropathic pain after spinal cord injury in rats. Experimental neurology. $2012 ; 236: 268-82$.

12. Tu WZ, Li SS, Jiang X, Qian XR, Yang GH, Gu PP, et al. Effect of electro-acupuncture on the BDNF-TrkB pathway in the spinal cord of CCI rats. International journal of molecular medicine. $2018 ; 41: 3307-15$.

13. Jiang YL, Yin XH, Shen YF, He XF, Fang JQ. Low Frequency Electroacupuncture Alleviated Spinal Nerve Ligation Induced Mechanical Allodynia by Inhibiting TRPV1 Upregulation in Ipsilateral Undamaged Dorsal Root Ganglia in Rats. Evidencebased complementary and alternative medicine : eCAM. 2013 ; $2013: 170910$

14. Dong ZQ, Ma F, Xie H, Wang YQ, Wu GC. Changes of expression of glial cell line-derived neurotrophic factor and its receptor in dorsal root ganglions and spinal dorsal horn during electroacupuncture treatment in neuropathic pain rats. Neuroscience letters. $2005 ; 376: 143-8$.

15. Dong ZQ, Xie H, Ma F, Li WM, Wang YQ, Wu GC. Effects of electroacupuncture on expression of somatostatin and preprosomatostatin mRNA in dorsal root ganglions and spinal dorsal horn in neuropathic pain rats. Neuroscience letters. 2005 ; 385 : 189-94. 


\section{ACUPUNCTURE}

16. Kim SH, Chung JM. An experimental model for peripheral neuropathy produced by segmental spinal nerve ligation in the rat. Pain. $1992 ; 50: 355-63$.

17. Li WM, Cui KM, Li N, Gu QB, Schwarz W, Ding GH, et al. Analgesic effect of electroacupuncture on complete Freund's adjuvant-induced inflammatory pain in mice: a model of antipain treatment by acupuncture in mice. The Japanese journal of physiology. $2005 ; 55: 339-44$.

18. Tanga FY, Raghavendra V, DeLeo JA. Quantitative real-time RT-PCR assessment of spinal microglial and astrocytic activation markers in a rat model of neuropathic pain. Neurochem Int. $2004 ; 45: 397-407$.

19. Clark AK, Gentry C, Bradbury EJ, McMahon SB, Malcangio M. Role of spinal microglia in rat models of peripheral nerve injury and inflammation. European journal of pain (London, England). $2007 ; 11: 223-30$.

20. Clark AK, Yip PK, Grist J, Gentry C, Staniland AA, Marchand F, et al. Inhibition of spinal microglial cathepsin $S$ for the reversal of neuropathic pain. Proceedings of the National Academy of
Effects of Electroacupuncture on the Expression of CCL2

Sciences of the United States of America. 2007 ; 104 : 10655-60.

21. MikaJ, Zychowska M, Popiolek-Barczyk K, Rojewska E, Przewlocka B. Importance of glial activation in neuropathic pain. European journal of pharmacology. $2013 ; 716: 106-19$.

22. Tsuda M, Shigemoto-Mogami Y, Koizumi S, Mizokoshi A, Kohsaka S, Salter MW, et al. P2X4 receptors induced in spinal microglia gate tactile allodynia after nerve injury. Nature. 2003 ; 424 : 77883.

23. Verge GM, Milligan ED, Maier SF, Watkins LR, Naeve GS, Foster AC. Fractalkine (CX3CL1) and fractalkine receptor (CX3CR1) distribution in spinal cord and dorsal root ganglia under basal and neuropathic pain conditions. The European journal of neuroscience. $2004 ; 20: 1150-60$.

24. Zhuang ZY, Kawasaki Y, Tan PH, Wen YR, Huang J, Ji RR. Role of the CX3CR1/p38 MAPK pathway in spinal microglia for the development of neuropathic pain following nerve injury-induced cleavage of fractalkine. Brain, behavior, and immunity. 2007; 21 : 642-51. 